\title{
PET scan in a patient with pneumococcal sepsis
}

\author{
Tal Grenader $\cdot$ Linda Shavit
}

Received: 22 April 2011/Accepted: 24 May 2011/Published online: 10 June 2011

(C) SIMI 2011

A 63-year-old man presented to the Otolaryngology department with severe dysphagia. On laryngoscopy, a whitish nodule $3 \mathrm{~cm}$ in size exiting from the posterior commissurae of the larynx, and almost obstructing the hypopharyngeal space, was observed. The excision biopsy specimen was diagnosed as moderately differentiated squamous cell carcinoma of the hypopharynx. A staging procedure with positron emission tomography-computer tomography (PET-CT) was recommended.

The day before the procedure, the patient experienced chills, and his temperature increased up to $38.9^{\circ} \mathrm{C}$. He was evaluated in the emergency department (ED) of our hospital. The plain film of the chest X-ray study revealed a small infiltrate in the right lower lobe (arrows Fig. 1a, b) and the patient was discharged on oral antimicrobial therapy to manage his pneumonia. The patient's wife had been hospitalized with pneumococcal pneumonia 2 days earlier.

The day after his ED visit, the patient underwent the previously arranged ambulatory PET-CT that revealed a significant increase of fluorine-18-fluorodeoxyglucose (F18-FDG) uptake in the hypopharyngeal mass (thick arrow Fig. 1a), diffuse uptake in the bone marrow of the spine, ribs, and pelvis without underlying bone lesions on a computed tomography (CT scan) (black arrows Fig. 2a-c), and an increased uptake in the right lower lobe consolidation consistent with pneumonia (white arrows Fig. 2b, c). The diagnosis of pneumonia was supported by significant

T. Grenader $(\bowtie)$

Department of Oncology, Sha' are Zedek Medical Center, P.O. Box 3235, 91031 Jerusalem, Israel

e-mail: talgrenader65@hotmail.com

L. Shavit

Department of Internal Medicine,

Sha' are Zedek Medical Center, Jerusalem, Israel
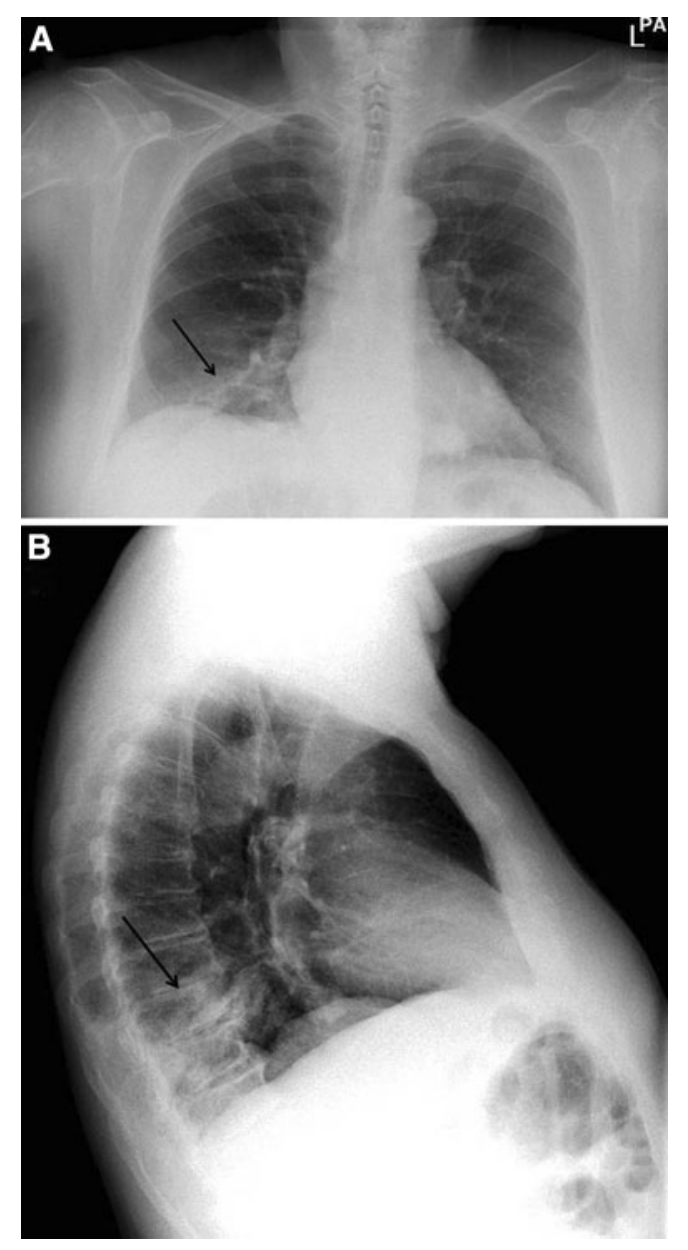

Fig. 1 The plain film of the chest X-ray study revealed a small infiltrate in the right lower lobe

leukocytosis with white blood cell count of $19.8 \times 10^{9} / \mathrm{L}$ and $92 \%$ neutrophils. The blood cultures grew Streptococcus pneumonia. The patient was treated with 
Fig. 2 A significant increase of F18FDG uptake in the hypopharyngeal mass (thick arrow a), diffuse uptake in the bone marrow of the spine, ribs and pelvis without underlying bone lesions on CT scan (black arrows $\mathbf{a}-\mathbf{c})$, and an increased uptake in a right lower lobe consolidation (white arrows $\mathbf{b}, \mathbf{c}$ )
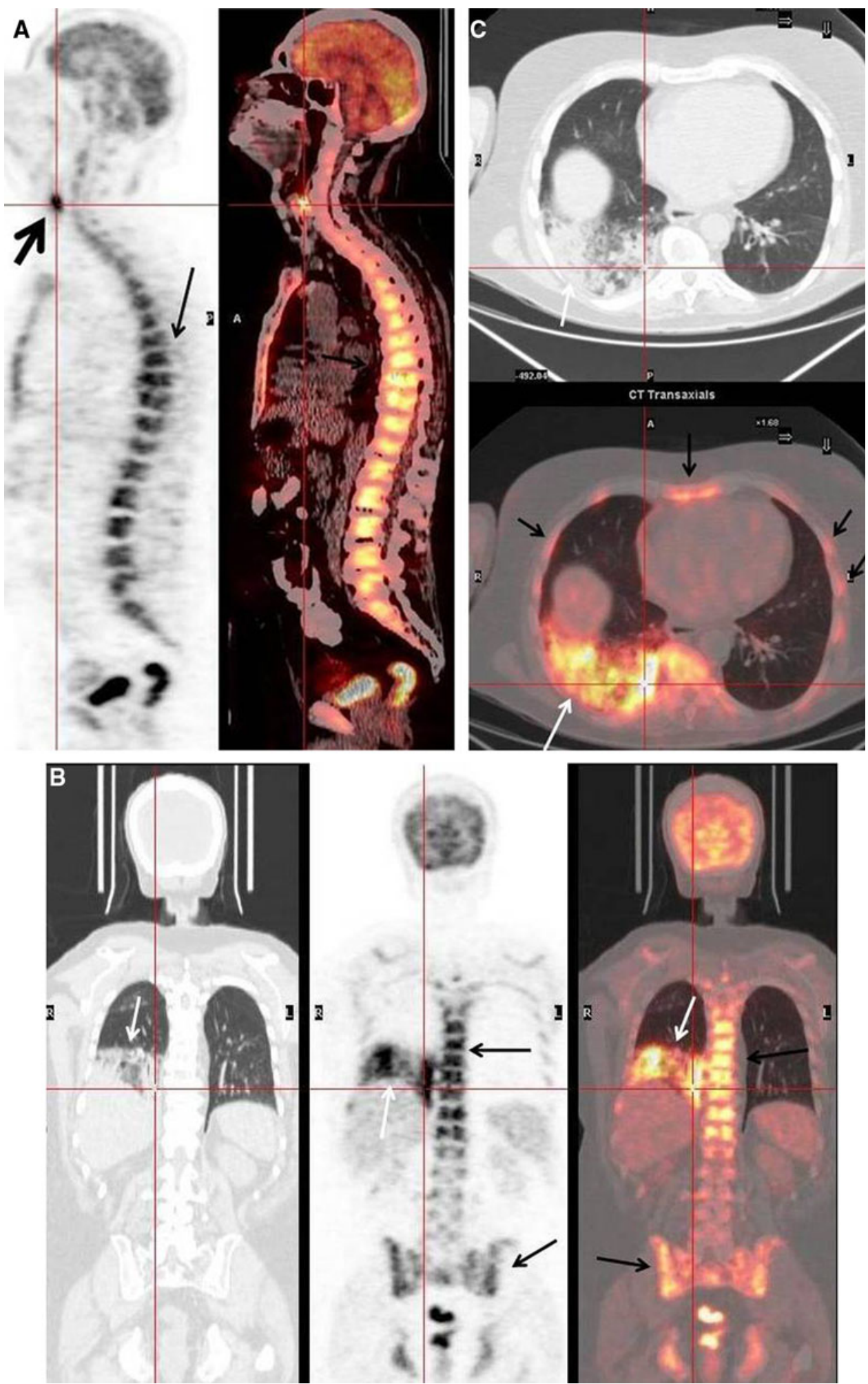

cefuroxime with subsequent improvement of symptoms, and a reduction of leukocytes and neutrophils to the normal range.

A diffuse increase in FDG uptake has been reported in hyperplastic bone marrow. With the increasing use of cytokine therapy and of FDG-PET for the assessment of the efficacy of chemotherapy, increased FDG uptake in normal bone marrow during and after cytokine therapy has often been observed [1]. Increased uptake in normal bone marrow during and after G-CSF or GM-CSF therapy, or 
during severe infection may mimic progressive bone or bone marrow metastases $[1,2]$.

Bone marrow activity increasing FDG uptake also has been reported in viral infections, causing severe immune response. Lustberg et al. report a case of acute EpsteinBarr virus (EBV) infection in which FDG-PET-CT shows avid FDG uptake in numerous abdominal/pelvic nodes, liver, spleen, and bone marrow. A bone marrow biopsy showed diffuse infiltration with plasmacytoid cells that were not kappa lambda restricted [3]. Increased FDG uptake in reactive lymph nodes and the spleen has also been reported in other systemic infections, including human immunodeficiency virus and hepatitis $C$ virus [4].

In our case, high accumulation of F18-FDG in the bone marrow is probably due to the appearance of bone marrow activity in response to severe pneumococcal infection. Diffuse uptake in the bone marrow of spine, ribs, and pelvis without underlying bone lesions on a CT scan makes the diagnosis of bone metastases unlikely in our patient.
Conflict of interest None.

\section{References}

1. Kazama T, Swanston N, Podoloff DA, Macapinlac HA (2005) Effect of colony-stimulating factor and conventional- or high-dose chemotherapy on FDG uptake in bone marrow. Eur J Nucl Med Mol Imaging 32:1406-1411

2. Sugawara Y, Fisher SJ, Zasadny KR, Kison PV, Baker LH, Wahl RL (1998) Preclinical and clinical studies of bone marrow uptake of fluorine-1-fluorodeoxyglucose with or without granulocyte colony-stimulating factor during chemotherapy. J Clin Oncol 16:173-180

3. Lustberg MB, Aras O, Meisenberg BR (2008) FDG PET/CT findings in acute adult mononucleosis mimicking malignant lymphoma. Eur J Haematol 81:154-156

4. Jacene HA, Stearns V, Wahl RL (2006) Lymphadenopathy resulting from acute hepatitis $\mathrm{C}$ infection mimicking metastatic breast carcinoma on FDG PET/CT. Clin Nucl Med 31:379-381 